\title{
AVALIAÇÃO DA CAPACIDADE FUNCIONAL DE IDOSOS COM DOENÇA RENAL CRÔNICA EM TRATAMENTO DE HEMODIÁLISE
}

\author{
EVALUATION FUNCTIONAL CAPACITY OF ELDERLY WITH \\ CHRONIC RENAL IN HEMODIALYSIS TREATMENT
}

\section{Fábio Pereira de Carvalho'; Igho Leonardo do Nascimento Carvalho²; Antônia Sylca de Jesus Sousa3; Camila Duarte Simões; ${ }^{4}$ Erisonval Saraiva da Silva ${ }^{5}$; Joelita de Alencar Fonseca Santos ${ }^{6}$.}

\section{RESUMO}

Objetivo: Avaliar a capacidade funcional em idosos com insuficiência renal crônica que realizam tratamento de hemodiálise. Metodologia: Estudo transversal, descritivo, realizado em Teresina$\mathrm{PI}$, no período de agosto a novembro de 2015, com amostra de 60 idosos. Aplicado questionário estruturado e a Medida de Independência Funcional (MIF), sendo obtido frequência simples e Teste Qui-quadrado de Pearson ( $p<0,05)$. Resultados: A maioria dos pacientes avaliados apresentou o grau de independência completa (28,3\%), embora a média total da MIF (104,3 pontos) indicou grau de dependência no nível de supervisão. 0 conhecimento sobre a patologia e renda apresentou relação estatisticamente significante com a capacidade funcional. Considerações finais: 0 tratamento de hemodiálise pouco interfere sobre a capacidade funcional dos idosos, mas sugere ações de saúde baseadas em atividades físicas e lazer, otimizando a manutenção da qualidade de vida.

Descritores: Atividades Cotidianas; Saúde do Idoso; Insuficiência Renal Crônica; Diálise Renal.

\begin{abstract}
Objective: To evaluate the functional capacity in elderly patients with chronic renal failure in hemodialysis treatment. Methodology: Transversal and descriptive study, carried out from August to November 2015 in Teresina city with sample of 60 elderly. It was applied a structured questionnaire and the Functional Independence Measure (FIM), obtaining simple frequency and chi-square test of Pearson $(p<0.05)$. Results: Most assisted patients had the degree of complete independence $(28.3 \%)$, although the total average of the FIM (104.3 points) indicated a degree of dependence in the level of supervision. Knowledge about the pathology and income showed a statistically significant relationship with the functional capacity. Final considerations: The hemodialysis treatment interferes in the functional capacity of the elderly, suggesting health interventions based on stimulus to practices of physical and leisure activities, enhancing the maintenance of the quality of life of individuals.
\end{abstract}

Descriptors: Activities of Daily Life; The Elderly Health; Chronic Renal Insufficiency; Renal Dialysis.
${ }^{1}$ Graduado em Enfermagem pela Universidade Federal do Piauí (UFPI), Floriano, PI, Brasil.

${ }^{2}$ Doutorando em Saúde Coletiva pela Universidade de Fortaleza (UNIFOR), Fortaleza, CE, Brasil.

${ }^{3}$ Graduada em Enfermagem pela Universidade Federal do Piauí (UFPI), Picos, Piauí, Brasil.

${ }^{4}$ Graduada em Enfermagem pela Faculdade Metropolitana da Grande Fortaleza (FAMETRO), Fortaleza, CE, Brasil.

${ }^{5}$ Mestre em Terapia Intensiva pela Sociedade Brasileira de Terapia intensiva (SOBRATI), Teresina, PI, Brasil.

${ }^{6}$ Mestre em Terapia Intensiva pela Universidade Brasileira de Terapia Intensiva (UNIBRATI), São Paulo, SP, Brasil. 


\section{Introdução}

A capacidade funcional ou capacidade de realizar as atividades de vida diária com autonomia e independência tem sido uma das dimensões de saúde considerada fundamental na avaliação da saúde da população idosa, principalmente daqueles com Doença Renal Crônica (DRC), devido ao potencial de comprometimento. Nesse sentido, existe 0 amplo reconhecimento que, para o idoso em tratamento hemodialítico, as alterações funcionais interferem de forma significativa no desenvolvimento das suas atividades de vida diária, requerendo intervenções de saúde ${ }^{1}$.

A DRC é a perda lenta, progressiva e irreversível das funções renais. Os rins possuem um processo adaptativo, permanecendo assintomático até que se tenha $50 \%$ da função renal comprometida. Quando diagnosticado a DRC, adota-se tratamento conservado baseado em medicamentos e dieta, embora somente quando a função renal está abaixo de 15 ou 10\% torna-se necessário o uso de métodos invasivos de tratamento, como diálise (hemodiálise ou diálise peritoneal) ou transplante renal'2.

Atualmente a hemodiálise é a forma de tratamento mais utilizada em idosos com IRC, baseada na circulação extracorpórea, feita entre membranas derivadas de celulose que atuam como membrana semipermeável. 0 paciente idoso com IRC em programa de hemodiálise necessita conviver com uma doença incurável e de tratamento doloroso que, devido sua evolução e complicações, gera limitações e impactos que afetam a vida desse indivíduo e de sua família-4.

Os pacientes idosos constituem a parcela da população com crescimento mais rápido em todo o mundo, inclusive no Brasil, e apresentam alta prevalência de DRC. Estes são particularmente susceptíveis à diminuição da função renal, devido ao declínio fisiológico da filtração glomerular (FG) relacionado à idade e comorbidades, tais como diabetes mellitus (DM) e hipertensão arterial sistêmica (HAS). Com isso, a DRC e seu tratamento acaba por interferir na vida dos idosos impedindo ou limitando suas atividades de vida diária, por causarem muitas vezes incapacidades físicas ou emocionais ${ }^{5-6}$.

A avaliação da capacidade funcional torna-se essencial para identificação precoce dos fatores de risco para incapacidade, estabelecimento de diagnóstico, prognóstico e julgamento clínico adequado, que nortearão as decisões sobre os tratamentos e cuidados necessários às pessoas idosas com IRC, especialmente os que realizam tratamento hemodialítico. Essa avaliação é de extrema importância porque contribui para formulação de um plano terapêutico individual, além de servir como parâmetro de comparação de intervenções de saúde e para qualificação de profissionais de saúde, melhorando sua prática assistencial ${ }^{7,8}$.

Para a equipe multidisciplinar, a avaliação da capacidade funcional do idoso em tratamento hemodialítico proporcionará um maior conhecimento sobre o impacto na autonomia do indivíduo. Dessa forma, o profissional terá a possibilidade de direcionar sua assistência, avaliando, elaborando e executando os cuidados prestados ao idoso, fornecendo o apoio necessário para preservação ou redução de danos à capacidade funcional.

O estudo ainda pretende otimizar a adaptação do idoso ao tratamento de hemodiálise, contribuindo para redução de condições que interfiram na capacidade de realizar suas atividades de vida diária à medida que subsidia estratégias de saúde que visem a manutenção da autonomia, independência e, principalmente, qualidade de vida. Por isso, o estudo objetiva avaliar a capacidade funcional em idosos com insuficiência renal crônica que realizam tratamento de hemodiálise.

\section{Metodologia}

Trata-se de estudo transversal, analítico, com abordagem quantitativa. $O$ estudo foi aprovado pelo Comitê de Ética em Pesquisa em Seres Humano da Universidade Federal do Piauí, Campus Amílcar Ferreira Sobral, sob o parecer n068959/2015 e Certificado de Apresentação para Apreciação Ética (CAAE) número 47351015.9.0000.5660

Realizado na clínica especializada em nefrologia, referência em tratamento em hemodiálise, localizada no município de Teresina, capital do estado do Piauí, com população estimada de 840.600 habitantes, sendo um centro referência para serviços de saúde no norte e nordeste brasileiro.

O estudo adotou uma amostra não probabilística, na qual participaram 60 idosos com diagnóstico de IRC que realizavam tratamento hemodialítico, durante período mínimo de três meses, devido ser o tempo necessário à estabilidade e evolução do quadro clínico. A coleta de dados foi realizada no período entre agosto a setembro de 2015 , exclusivamente pelo pesquisador assistente, acadêmico do curso de enfermagem da Universidade Federal do Piauí (UFPI). 
A coleta foi desenvolvida por meio de entrevista a partir de instrumento estruturado, contendo questionamentos que revelam características socioeconômicas, familiares e de saúde, bem como aplicação do instrumento multidimensional "Medida de Independência Funcional" (MIF), validado no Brasil e presente no Caderno de Atenção da Pessoa Idosa, que quantifica o grau de solicitação de cuidados de terceiros de que o portador de uma deficiência física necessita para a realização de atividades motoras e cognitivas, mas que pode ser em outras comorbidades, tais como DRC ${ }^{9}$.

Na MIF, quanto maior a pontuação obtida, maior a independência do paciente na realização de suas atividades de vida diária. A MIF total compreende dezoito atividades distribuídas em dois domínios. No motor, a ênfase é dada ao autocuidado, ao controle de esfíncter, à mobilidade e à locomoção, englobando treze atividades. No domínio cognitivo/ social compreende-se as funções de comunicação e cognição social, contendo cinco atividades. Considerando todas as atividades, a pontuação total pode variar entre 18 e 126, sendo dividida em sete níveis de dependência, abrangendo do nível 1 (ajuda total - menor que 31 pontos), nível 2 (ajuda máxima - entre 32 e 62 pontos), nível 3 (ajuda moderada - entre 63 e 93 pontos), nível 4 (ajuda com contato mínimo - a partir de 94 pontos), nível 5 (supervisão - entre 95 e 111 pontos), nível 6 (independência modificada - entre 112 e 125 pontos) e nível 7 (independência completa - igual a 126 pontos) ${ }^{10}$.

Os dados foram armazenados e analisados no pacote estatístico Statistical Package for the Social Sciences (SPSS) 19.0, sendo que o plano de análise compreendeu a estatística descritiva de avaliação da MIF e do estilo de vida por tabelas e gráficos. Em seguida, foi investigado o nível de significância a partir da classificação da MIF - variável dependente - com as variáveis sociodemográficas, familiar e de saúde por meio da aplicação do Teste Qui-quadrado, nível de significância $p<0,05$.

\section{Resultados}

Os achados mais significativos do estudo foram a manutenção do nível de independência funcional e preservação das atividade de vida diária, embora os participantes afirmaram precisar abandonar suas atividades de trabalho. A renda e o conhecimento apresentam relação estatisticamente significativa com a capacidade funcional.

A aplicação do instrumento MIF constatou a ocorrência de quase todos possíveis níveis de dependência com exceção do nível de ajuda total, demonstrando que ajuda máxima representa 6,7\%; ajuda moderada 13,3\%; ajuda com contato mínimo 18,3\%; supervisão 20,0\%; independência modificada 13,3\%; independência completa 28,3\% (Gráfico 01).

Gráfico 01 - Classificação da MIF para idosos com IRC em tratamento hemodialítico. Teresina-PI, 2015.

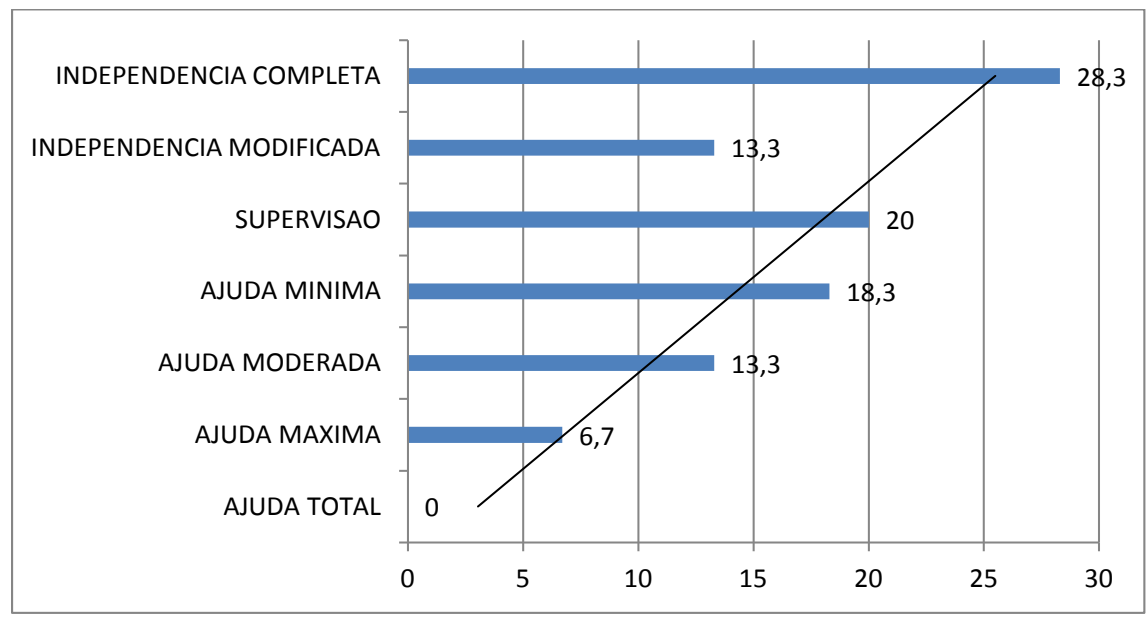

Verificou-se que a média total da MIF foi de 104,3 pontos, indicando um grau de dependência no nível de supervisão ou preparação. Quando analisados os resultados quanto aos subdomínios, o grau de dependência encontrado correspondeu às seguintes médias: função autocuidado 38,6; função controle de esfíncter 12,6; mobilidade 16,9; locomoção 6,5 e cognição 29,4 (Tabela 01). 
Tabela 01 - Avaliação da capacidade funcional dos pacientes idosos que realizam tratamento de hemodiálise segundo os subdomínios da Medida de Incapacidade Funcional. Teresina-PI, 2015.

\begin{tabular}{|c|c|c|c|c|}
\hline SUBDOMINIO & & & & \\
\hline Autocuidado & 10 & 42 & 38,6 & 6,7 \\
\hline Controle dos Esfíncteres & 02 & 14 & 12,6 & 3,2 \\
\hline Mobilidade & 03 & 21 & 16,9 & 6,1 \\
\hline Locomoção & 02 & 14 & 6,5 & 4,9 \\
\hline Cognição & 15 & 35 & 29,4 & 4,7 \\
\hline TOTAL (MIF) & 37 & 126 & 104,3 & 20,8 \\
\hline
\end{tabular}

Os hábitos de vida dos pacientes idosos que realizam tratamento de hemodiálise demonstram que $76,7 \%$ dos idosos conseguem desenvolver suas atividades de vida diária de forma autônoma e independente. Quanto à atividade de vida que o idoso teve que abandonar, o trabalho foi atividade mais citada por 58,3\% dos participantes. Quando questionados sobre a realização de atividade física no cotidiano, somente 13,3\% afirmaram praticar atividade física, sendo que a atividade mais praticada é a caminhada (Tabela 02).

Tabela 02 - Hábitos de vida dos pacientes idosos que realizam tratamento de hemodiálise. Teresina-PI, 2015.

\begin{tabular}{c|c|c}
\hline VARIÁVEL & Frequência absoluta & Frequên. Relativa (\%) \\
\hline \multicolumn{3}{c}{ Consegue desenvolver suas atividades de vida diária } \\
com autonomia e independência (n= 60)
\end{tabular}

Analisando a evolução das diferentes áreas, observamos que as variáveis renda e conhecimento são estatisticamente significativas na classificação da MIF, pois apresentam valor menor ou igual a 0,05 , mostrando assim sua relação direta com perda da independência funcional dos pacientes em tratamento de hemodiálise no presente estudo. Quanto às demais os dados mostram seu baixo valor significativo em relação à MIF (Tabela 03). 
Verificou-se, através da analise dos dados, que na relação de dependência entre as características socioeconômicas e de saúde com a MIF, dentre a população em estudo, que os indivíduos do sexo masculino têm nível satisfatório de escolaridade; renda maior que três salários mínimos; conseguem realizar suas atividades de vida diária; não apresentaram intercorrências durante a hemodiálise; tem maior conhecimento sobre a patologia e que o tratamento interfere no convívio social ou familiar, mas que durante o procedimento mantêm a independência funcional.

Quanto aos indivíduos que se mostraram dependentes funcionalmente estão os idosos do sexo feminino, que demonstram ter nível de escolaridade baixo; recebem menos de três salários mínimos; não conseguem realizar suas atividades de vida diária; apresentaram alguma intercorrência durante a hemodiálise; não têm conhecimento sobre a patologia e relataram não ter interferência no convívio social e familiar durante o tratamento.

Tabela 03 - Relação entre características socioeconômicas e de saúde dos pacientes idosos que realizam tratamento de hemodiálise com a MIF. Teresina-PI, 2015.

\begin{tabular}{|c|c|c|c|c|c|}
\hline VARIÁVEL & $\mathbf{p}$ & \multicolumn{2}{|c|}{ INDEPENDÊNCIA } & \multicolumn{2}{|c|}{ DEPENDÊNCIA } \\
\hline Sexo & 0,53 & & & & \\
\hline Masculino & & 15 & 35,7 & 27 & 64,3 \\
\hline Feminino & & 02 & 11,1 & 16 & 88,9 \\
\hline Escolaridade & 0,14 & & & & \\
\hline Baixa & & 08 & 21,6 & 29 & 78,4 \\
\hline Satisfatória & & 09 & 39,1 & 14 & 60,9 \\
\hline Renda & 0,02 & & & & \\
\hline Até $3 \mathrm{SM}^{*}$ & & 13 & 29,5 & 41 & 70,5 \\
\hline Acima de $3 \mathrm{SM}^{*}$ & & 04 & 66,6 & 02 & 33,4 \\
\hline Atividade de vida diária & 0,07 & & & & \\
\hline Sim & & 17 & 36,9 & 29 & 63,1 \\
\hline Não & & - & & 14 & 100,0 \\
\hline Intercorrência & 0,10 & & & & \\
\hline Sim & & 09 & 21,9 & 32 & 78,9 \\
\hline Não & & 08 & 72,7 & 11 & 27,7 \\
\hline Conhecimento & 0,02 & & & & \\
\hline Sim & & 07 & 53,8 & 06 & 46,2 \\
\hline Não & & 10 & 21,2 & 37 & 78,8 \\
\hline Relações familiares & 0,77 & & & & \\
\hline Sim & & 02 & 33,4 & 04 & 66,6 \\
\hline Não & & 15 & 27,8 & 39 & 72,2 \\
\hline Relações sociais & 0,84 & & & & \\
\hline Sim & & 0,1 & 33,4 & 02 & 66,6 \\
\hline Não & & 16 & 28,0 & 41 & 72,0 \\
\hline
\end{tabular}

*Salário mínimo no período da coleta $\mathrm{R} \$$ 788,00 reais. 


\section{Discussão}

A avaliação funcional é conceituada como a designação dada para uma função específica, a capacidade de realizar autocuidado e atender suas necessidades, ou seja, as atividades de vida diária (AVD). A MIF mensura capacidade funcional e independência, estimando o grau de dificuldade ou limitações atribuídas a cada pessoa ${ }^{11}$.

Neste estudo, a maioria dos pacientes avaliados apresentou o grau de independência completa na classificação geral da MIF, demonstrando assim estarem completamente aptos a realizarem suas atividades sem a necessidade de maiores cuidados de terceiros. Isso implica que, apesar de todos os obstáculos e limitações impostos aos idosos que realizam o tratamento hemodialítico, a maior parcela deles consegue fazer suas atividades do dia a dia sem a necessidade do auxílio de terceiros, realizando tarefas significativas para o seu bem estar, apesar da interferência da idade na capacidade funcional.

Outra classificação representativa foi o nível de dependência de supervisão, na qual os idosos em tratamento hemodialítico apresentam necessidade de acompanhamento de terceiros para realizar suas atividades diárias com segurança. Esse nível se refere a dependência modificada em que existe apenas a necessidade da presença física, sem contato para incentivar, ajudar ou preparar itens que possam satisfazer as necessidades básicas desses indivíduos ${ }^{8}$.

Quanto aos subdomínios da MIF, os que menos apresentaram comprometimento e dependência foram a locomoção, controle dos esfíncteres e mobilidade. Demonstrando que os pacientes idosos em tratamento hemodialítico apresentam bom desempenho em atividades como caminhar, subir e descer escadas, mudar de um local para outro e realizar suas atividades fisiológicas sem a necessidade da ajuda de terceiros.

O presente estudo corrobora com pesquisas nas quais fatores como anúria, diminuição do débito urinário, uso de medicações para controle da função intestinal proporcionam a esses subdomínio pouca influência na capacidade do idoso realizar suas atividades diárias. Quanto à locomoção e mobilidade, os idosos em hemodiálise não demonstram dificuldade, corroborando com outros estudos que aplicaram a MIF em pacientes que utilizam esta terapêutica ${ }^{12-13}$.

Os subdomínios mais afetados em sua capacidade funcional devido ao tratamento hemodialítico nos idosos foram o autocuidado e a cognição, demonstrado ter uma relação direta com a diminuição da independência funcional.

Acredita-se ainda que a maior frequência de idosos com comprometimento no autocuidado pode estar ligado à variável idade, pois constatou-se que com o aumento da idade há um declínio na capacidade para 0 autocuidado $\mathrm{e}$ na independência funcional. Logo, conclui-se que a idade, associada com as dificuldades impostas pela hemodiálise, dificulta 0 autocuidado desses pacientes ${ }^{14}$.

A alta frequência de idosos com dependência associada a fatores cognitivos pode estar relacionada ao alto número de idosos com baixa escolaridade. Foi observado que o baixo desempenho cognitivo aliado a sua patologia era favorecido quando os idosos, em sua maioria, tinham, no máximo, quatro anos de escolaridade.

Os idosos com nível de escolaridade baixo e em um tratamento que exige muito de sua capacidade psicológica têm seu desempenho cognitivo reduzido, necessitando da ajuda de terceiros para desenvolver suas atividades diárias ${ }^{15}$.

A aplicabilidade da MIF como instrumento de avaliação funcional em pacientes idosos em tratamento de IRC, mostrou-se ser uma ferramenta norteadora para os serviços de referência e para os profissionais de saúde. Dessa forma estes poderão orientar suas intervenções no tratamento adequado a estes pacientes, melhorando sua recuperação e contribuindo para o desenvolvimento de medidas de prevenção e manutenção da independência funcional destes.

Durante a pesquisa contatou-se a incoerência de opinião dos pesquisados, pois a maioria dos idosos revelou ter plena capacidade para realizar suas atividades de vida diária, mesmo em tratamento hemodialítico, ao mesmo tempo em que uma grande parcela dos entrevistados revelou abandono e interferência nas suas atividades laborais, devido ao tratamento.

A variável idade pode interferir na independência desse paciente, implicando sobre a necessidade de cuidados e comprometimento da rotina de trabalho ${ }^{16}$. As atividades laborais nos idosos fazem parte não apenas de uma forma de obter rendimentos financeiros, mas também de manter sua autoestima, sua independência cognitiva, sua participação efetiva perante a sociedade, sendo uma importante atividade desenvolvida no dia a dia.

Quando o idoso tem seu processo de trabalho interrompido por conta do tratamento de uma doença, gera-se um declínio não somente financeiro, mas também psicossocial neste paciente, fato esse que acaba potencializando seu estado de enfermo de uma forma geral. Nesse contexto, tornam-se necessárias medidas que priorizem a inserção dos idosos em tratamento hemodialítico em atividades que proporcionem seu envelhecimento ativo, melhorando assim sua qualidade de vida e, consequentemente, promovendo a independência funcional de terceiros. 
Ademais, as limitações impostas pelo tratamento impossibilitam que estes indivíduos mantenham uma jornada de trabalho adequada, que ocasiona baixa renda e representa uma condição de risco para saúde mental e física desses pacientes, bem como interferindo na dinâmica, na rotina e no lazer ${ }^{17}$.

A baixa escolaridade é refletida no pouco conhecimento sobre a doença dificultando assim ações de promoção da saúde e manutenção da capacidade funcional dos idosos em hemodiálise. Além disso dificulta adesão do paciente ao regime terapêutico e limita seu acesso a informações, o que contribui para sua dependência na realização de suas funções diárias ${ }^{14}$.

Quando se trata do sexo do indivíduo, os achados da pesquisa contrariam os dados de outras pesquisas disponibilizadas. Em idosos, a variável sexo está fortemente associado a ocorrência de dependência, sendo duas vezes maior a chance para idosos do sexo feminino em relação aos do sexo masculino ${ }^{18}$.

Considerando as dimensões físicas, houve uma piora na qualidade de vida dos idosos associada com o aumento da idade; sexo feminino; relato de duas ou mais internações no último ano e presença de três ou mais doenças crônicas auto referidas ${ }^{16}$. Nesse sentido, foi observado 0 aumento das intercorrências, ocasionado pelo tratamento e pelas comorbidades, pois diminuem o grau de independência funcional dos idosos.

Renda e conhecimento sobre a patologia, além de variáveis como sexo, escolaridade e intercorrência, relacionados ao tratamento hemodialítico nos idosos possuem forte influência sobre a independência funcional desses pacientes. A baixa escolaridade, pouco conhecimento e grande número de intercorrências relacionadas, principalmente comorbidades e idade avançada, aumentam a dificuldade de prevenção, promoção e manutenção da independência funcional e qualidade de vida dessa população'.

Cabe ressaltar que a dependência nas atividades de vida diária é fator que pode ser mutável com prevenção e reabilitação e, embora os principais conceitos de prevenção em saúde estejam assimilados pelos profissionais da área, percebe-se muita dificuldade na operacionalização, principalmente, quando se refere a grupos de pacientes crônicos, como aqueles com IRC ${ }^{13-14}$.

A avaliação da independência funcional constitui importante ferramenta norteadora de ações de saúde que compõem o plano terapêutico dos idosos em tratamento hemodialítico. O planejamento de ações de saúde, a partir dessa avaliação, direciona e prioriza a assistência do indivíduo idoso segundo a concepção holística e de promoção da qualidade de vida.

\section{Considerações Finais}

O estudo de avaliação da capacidade de idosos em tratamento hemodialítico permitiu identificar influência do tratamento na capacidade funcional, especialmente o baixo nível de escolaridade, o conhecimento sobre a patologia e a renda menor que três salários mínimos. Os idosos participantes do estudo mantiveram a independência funcional ou dependência supervisionada, referente a comprometimento na capacidade cognitiva e de autocuidado. Com isso, observa-se que a falta de conhecimento sobre a sua doença associado ao baixo poder aquisitivo dos pacientes idosos em tratamento hemodialítico são fatores que contribuem para o declínio funcional dos idosos, devido afetar capacidade cognitiva e de autocuidado.

Os achados fornecem aos profissionais de saúde da equipe multiprofissional, responsável pela assistência ao idoso em tratamento hemodialítico, informações relevantes para o planejamento de ações direcionadas à manutenção da capacidade funcional desses idosos, o que contribui para o desenvolvimento de plano terapêutico numa concepção integral e holística do idoso.

Acredita-se que as intervenções de ações direcionadas à preservação da capacidade funcional subsidiarão a redução da interferência do tratamento hemodialítico e o reconhecimento da potência de tecnologias leves no contexto da assistência especializada em saúde.

Uma limitação desta pesquisa é o tipo de estudo (transversal), no qual o instrumento MIF foi aplicado uma única vez durante as sessões de hemodiálise, não sendo possível captar alterações ao longo de um período de realização rotineira de hemodiálise. Apesar do amplo uso da MIF, trata-se de medida autorreferida que expressa o contexto situacional momentâneo que, por sua vez, pode superestimar sua capacidade funcional.

Torna-se necessário ampliar as análises das relações entre capacidade funcional e as demais variáveis sobre a influência das ações de acompanhamento e orientação dos idosos, assim como na capacitação dos profissionais de saúde envolvidos no processo em busca da promoção e manutenção dos cuidados dessa população.

O estudo buscou esclarecer a importância da independência funcional para os idosos com IRC em tratamento de hemodiálise e as implicações desse conhecimento para o plano terapêutico e qualidade de vida desses idosos. Nesse sentido, o estudo ainda proporcionou um enriquecimento para o conhecimento científico, tendo como expectativas o subsídio paraa assistência de enfermagem e demais profissionais de saúde no estabelecimento de uma prática clínica cada vez mais qualificada, integral e humana. 


\section{Agradecimentos}

À Universidade Federal do Piauí-UFPI/Campus Amílcar Ferreira Sobral (CAFS)

À Clínica de Nefrologia - CLINEFRO

\section{Referências}

1. Oller G. Independência funcional e capacidade para o auto cuidado de pacientes em tratamento Hemodialítico. Ribeirão Preto: [s.n], 2012. Dissertação (Mestrado). Universidade de São Paulo. Escola de Enfermagem de Ribeirão Preto, 2012. 2. Sociedade Brasileria de Nefrologia. Insuficiência Renal. São Paulo. 2015. Disponível em: <http://www.sbn.org.br/ publico/insuficiencia-renal>. Acesso em 20 Abr. 2015a.

3. Higa K, Kost MT, Soares GM. Qualidade de vida de portadores de insuficiência renal crônica em tratamento de hemodiálise. Actual. Paul. Enferm. 2008; 21: 204-6.

4. Fassbinder TRC, Wilkemann ER, Schneider J, Wendland J, Oliveira OB. Capacidade funcional e qualidade de vida de pacientes com doença renal crônica pré-dialítica e em hemodiálise-Um estudo transversal. J. Bras. Nefrol. 2015; 37(1): $47-56$.

5. Bastos MG, Abreu PF. Doença Renal em Pacientes Idosos. J.Bras.Nefrol. 2009; 31: 59-65.

6. Kusumota L, Marques S, Haas VJ; Rodrigues RAP. Adultos e idosos em hemodiálise: avaliação da qualidade de vida relacionada à saúde. Acta paul. enferm. 2008; 21: 152-159.

7. BRASIL, Ministério da Saúde. Departamento de Atenção Básica. Envelhecimento e Saúde da pessoa idosa. Caderno n.19. Brasília: 2006.

8. Fialho CB, Lima-Costa MF, Giacomin KC, Loyola Filho, Al. Capacidade funcional e uso de serviços de saúde por idosos da Região Metropolitana de Belo Horizonte, Minas Gerais, Brasil: um estudo de base populacional. Cad. Saúde Pública. 2014; 30 (3): 599-610.

9. Riberto M, Miyazaki MH, Filho DJ, Sakamoto H; Battistella LR. Reprodutividade da versão brasileira da medida da independência funcional. Acta Fisiátrica. 2011; 8 (1): 45-52.

10.Itami LT. Causas Externas e seu Impacto sobre a Independência Funcional em Adultos com Fraturas. São Paulo: [s.n.], 2008. Dissertação (Mestrado) - Universidade de São Paulo. Escola de Enfermagem, 2008.

11. Silveira L, Macagnan JBA, Fuck JAB, Lagana MTC. Medida de independência funcional: um desafio para a enfermagem. Revista de Saúde Pública de Santa Catarina. 2011; 4 (1): 70-81.

12. Mortari DM, Scapini KB, Menta M, Rockemback CWF, Duarte A, Leguisano CP. Independência funcional de indivíduos com doença renal crônica terminal submetidos à hemodiálise. Revista Inspirar. 2009; 1 (3): 39-48.

13. Riella MC. Princípios da nefrologia e distúrbios hidroeletrolíticos. $5^{a}$ ed. Rio de Janeiro: Guanabara Koogan; 2010.

14. Oller GASAO, Ribeiro RCHM, Travagim DAS, Batista MA, Marques S, Kusumota L. Independência funcional em pacientes com doença renal crônica em tratamento hemodialítico. Rev. Latino-Am. Enfermagem. 2012; 20 (6): 1033-1040.

15.Talmelli LFS. Nível de independência funcional de idosos com doença de Alzheimer. Ribeirão Preto: [s.n.], 2009. Dissertação (Mestrado). Universidade de São Paulo. Escola de Enfermagem de Ribeirão Preto, 2009.

16.Braga SFM, Peixoto SV, Gomes IC, Acúrcio FA, Andrade ELG, Cherchiglia ML. Fatores associados com a qualidade de vida relacionada à saúde de idosos em hemodiálise. Rev. Saúde Pública. 2011; 45 (6): 1127-1136.

17. Dutra MC, Uliano EJM, Machado DFGP, Martins M, Trevisol FS, Trevisol DJ. Avaliação da função renal em idosos: um estudo de base populacional. J. Bras. Nefrol. 2014; 36 (3): 297-303.

18. Oliveira Junior HM, Formiga FFC, Alexandre, CS. Perfil clínico-epidemiológico dos pacientes em programa crônico de hemodiálise em João Pessoa - PB. J. Bras. Nefrol. 2014; 36 (3): 367-374. 


\section{Fábio Pereira de Carvalho}

Endereço para correspondência - Quadra: A, $n^{\circ} 27$,

Bairro: Res. Eplanada, CEP: 64040762, Teresina, PI, Brasil.

E-mail: fab2carvalho@hotmail.com

Lattes: http://lattes.cnpq.br/6504241286307701

Igho Leonardo do Nascimento Carvalho - igho_leonardo@yahoo.com.br

Antônia Sylca de Jesus Sousa - sylcasousa88@hotmail.com

Camila Duarte Simões - camilasimoesduarte@hotmail.com

Erisonval Saraiva da Silva - erisonval@gmail.com

Joelita de Alencar Fonseca Santos - joelitaalencar@hotmail.com

\section{Enviado em 21 de março de 2016.} Aceito em 18 de maio de 2016. 
